\title{
Quantitative Upright-Supine High-Speed SPECT Myocardial Perfusion Imaging for Detection of Coronary Artery Disease: Correlation with Invasive Coronary Angiography
}

\author{
Ryo Nakazato*$^{* 1-3}$, Balaji K. Tamarappoo*1-3, Xingping Kang ${ }^{1-3}$, Arik Wolak ${ }^{1-3}$, Faith Kite ${ }^{1}$, Sean W. Hayes ${ }^{1-4}$, \\ Louise E.J. Thomson ${ }^{1-4}$, John D. Friedman ${ }^{1-4}$, Daniel S. Berman ${ }^{1-4}$, and Piotr J. Slomka ${ }^{1-4}$ \\ ${ }^{1}$ Division of Nuclear Medicine, Department of Imaging, Cedars-Sinai Medical Center, Los Angeles, California; ${ }^{2}$ Division of \\ Cardiology, Department of Medicine, Cedars-Sinai Medical Center, Los Angeles, California; ${ }^{3}$ Cedars-Sinai Heart Institute, Cedars- \\ Sinai Medical Center, Los Angeles, California; and ${ }^{4}$ Department of Medicine, University of California at Los Angeles, School of \\ Medicine, Los Angeles, California
}

A recently developed camera system for high-speed SPECT (HS-SPECT) myocardial perfusion imaging shows excellent correlation with conventional SPECT. Our goal was to test the diagnostic accuracy of an automated quantification of combined upright and supine myocardial SPECT for detection of coronary artery disease $(\mathrm{CAD})(\geq 70 \%$ luminal diameter stenosis or, in left main coronary artery, $\geq 50 \%$ luminal diameter stenosis) in comparison to invasive coronary angiography (ICA). Methods: We studied 142 patients undergoing upright and supine HS-SPECT, including 56 consecutive patients $(63 \%$ men; mean age $\pm S D, 64 \pm 13$ y; $45 \%$ exercise stress) without known CAD who underwent diagnostic ICA within 6 mo of HSSPECT and 86 consecutive patients with a low likelihood of CAD. Reference limits for upright and supine HS-SPECT were created from studies of patients with a low likelihood of CAD. Automated software adopted from supine-prone analysis was used to quantify the severity and extent of perfusion abnormality and was expressed as total perfusion deficit (TPD). TPD was obtained for upright (U-TPD), supine (S-TPD), and combined upright-supine acquisitions (C-TPD). Stress U-TPD $\geq 5 \%$, S-TPD $\geq 5 \%$, and C-TPD $\geq 3 \%$ myocardium were considered abnormal for per-patient analysis, and U-TPD, S-TPD, and C-TPD $\geq 2 \%$ in each coronary artery territory were considered abnormal for per-vessel analysis. Results: On a per-patient basis, the sensitivity was $91 \%, 88 \%$, and $94 \%$ for U-TPD, S-TPD, and C-TPD, respectively, and specificity was 59\%, 73\%, and $86 \%$ for U-TPD, S-TPD, and C-TPD, respectively. C-TPD had a larger area under the receiver-operating-characteristic curve than U-TPD or S-TPD for identification of stenosis $\geq$ $70 \%$ (0.94 vs. 0.88 and $0.89, P<0.05$ and not significant, respectively). On a per-vessel basis, the sensitivity was $67 \%$, $66 \%$, and $69 \%$ for U-TPD, S-TPD, and C-TPD, respectively, and specificity was $91 \%, 94 \%$, and $97 \%$ for U-TPD, S-TPD, and C-TPD, respectively ( $P=0.02$ for specificity U-TPD vs. C-TPD). Conclusion: In this first comparison of HS-SPECT with ICA, new automated quantification of combined upright and

Received May 5, 2010; revision accepted Aug. 11, 2010.

For correspondence contact: Piotr Slomka, Division of Nuclear Medicine, Department of Imaging, Cedars-Sinai Medical Center, 8700 Beverly Blvd., Taper Building, Suite A047, Los Angeles, CA 90048.

E-mail: slomkap@cshs.org

${ }^{*}$ Contributed equally to this work.

COPYRIGHT @ 2010 by the Society of Nuclear Medicine, Inc. supine HS-SPECT shows high diagnostic accuracy for detecting clinically significant CAD, with findings comparable to those reported using conventional SPECT.

Key Words: high-speed SPECT; myocardial perfusion imaging; quantification; upright-supine; coronary artery disease

J Nucl Med 2010; 51:1724-1731

DOI: 10.2967/jnumed.110.078782

O everal dedicated hardware camera systems with optimized acquisition geometry, collimator design, and associated reconstruction software have been recently introduced by various vendors (Cardius XPO [Digirad, Inc.]; CardiArc [CardiArc]; and D-SPECT [Spectrum-Dynamics]) (1). The D-SPECT, for high-speed SPECT (HS-SPECT) using cadmium-zinc-telluride $(\mathrm{CZT})$ detectors and rotating detector columns (2-4), has been validated by comparison to conventional Anger camera imaging for rest-stress myocardial perfusion SPECT (MPS) $(3,5)$. This technology has been shown to provide an 8- to 10-fold increase in sensitivity, coupled with a 2-fold improvement in spatial resolution, enabling a significant reduction in imaging time and dose of radiopharmaceutical $(1,2,4)$. In a recent multicenter clinical trial, HS-SPECT has been shown to correlate well with conventional SPECT for rest-stress myocardial perfusion and function (5). However, to date, there have been no studies comparing the diagnostic accuracy of HS-SPECT for detecting physiologically significant coronary artery disease (CAD) with invasive coronary angiography (ICA).

We have previously developed and validated a quantification scheme in which reference limits are derived from patients with a low likelihood (LLk) of CAD without requiring optimization of regional thresholds by expert visual scoring (6). We have additionally implemented and validated an automated approach for quantification of MPS by integrating datasets acquired in both supine and prone positions (7). Unlike conventional MPS, HS-SPECT is performed with 
the patient in a semirecumbent upright position and, if desired, in the supine position. In this study, we sought to develop an automated approach to quantify total perfusion deficit (TPD), a computer-derived measure of the extent and severity of a perfusion defect, for combined upright-supine MPS using HS-SPECT and to assess the diagnostic accuracy of combined upright-supine quantification as compared with upright-alone or supine-alone quantification for the detection of hemodynamically significant $\mathrm{CAD}$ as defined by ICA in patients without a previous history of CAD.

\section{MATERIALS AND METHODS}

\section{Total Study Population}

The total study population consisted of 142 patients who underwent rest-stress HS-SPECT with rest-stress ${ }^{99 \mathrm{~m} T c-s e s t a m i b i}$ (5) or stress ${ }^{201} \mathrm{Tl}-$ rest ${ }^{99 \mathrm{~m}} \mathrm{Tc}$-sestamibi MPS (8) performed in both upright and supine positions at the Cedars-Sinai Medical Center. All patients provided written informed consent for the use of their clinical and imaging data for research purposes.

Angiographic Validation. For comparison of HS-SPECT with ICA, we included 56 consecutive patients ( 35 men, 21 women) who had ICA within 6 mo of rest-stress HS-SPECT after rest-stress ${ }^{99 \mathrm{~m} T c-s e s t a m i b i}(n=39)$ or stress ${ }^{201} \mathrm{Tl}-$ rest ${ }^{99 \mathrm{~m}} \mathrm{Tc}$-sestamibi ( $n=17$ ) MPS. The included patients had no prior myocardial infarction or coronary revascularization, nonischemic cardiomyopathy or valvular heart disease, or change in symptoms between MPS and ICA (Table 1$)$. The mean age ( \pm SD) was $64 \pm 13 \mathrm{y}$, and mean body mass index was $28 \pm 7 \mathrm{~kg} / \mathrm{m}^{2}$ (range, $19-53 \mathrm{~kg} / \mathrm{m}^{2}$ ). Four patients had typical angina, 31 had atypical angina, and 5 had shortness of breath. Exercise stress was used in $25(45 \%)$ patients, all of whom achieved the maximal target heart rate during exercise. An ischemic electrocardiogram response was noted in 17 patients who underwent exercise stress and in 2 patients who underwent vasodilator stress. The average time between HS-SPECT and ICA was $23 \pm 30 \mathrm{~d}$.

Creation of Reference Limits. For generation of reference limits for perfusion by HS-SPECT, we included 86 patients who had an LLk of CAD $(<15 \%)$ based on age, sex, symptoms, coronary risk factor, and the results of treadmill stress testing at the time of MPS
(9): rest-stress ${ }^{99 \mathrm{~m}} \mathrm{Tc}-$ sestamibi $(n=60)$ or stress ${ }^{201} \mathrm{Tl}$-rest ${ }^{99 \mathrm{~m}} \mathrm{Tc}-$ sestamibi $(n=26)$. Patients did not have angina, shortness of breath, an abnormal resting electrocardiogram, or an abnormal stress electrocardiogram response. Additionally, as per previous practice in generating reference limits $(6)$, the patients had normal rest and poststress HS-SPECT findings on visual assessment of upright and supine images (visual summed score $<3$ ). In the rest-stress ${ }^{99 \mathrm{~m}} \mathrm{Tc}-$ sestamibi population, sex-specific reference limits for both upright and supine acquisitions were derived from a group of 60 patients (30 men, 30 women) with an LLk of CAD. In the stress ${ }^{201}$ Tl-rest ${ }^{99 \mathrm{~m} T c-s e s t a m i b i}$ population, sex-specific reference limits for both upright and supine acquisitions were derived from a group of 26 patients (15 men, 11 women) with an LLk of CAD (Table 2).

\section{Imaging and Stress Protocols}

Patients were instructed to abstain from any products containing caffeine for $24 \mathrm{~h}$ before the test. $\beta$-blockers and calciumchannel antagonists were terminated $48 \mathrm{~h}$ before testing, and nitrates were withheld for at least $6 \mathrm{~h}$ before testing. Stress testing was performed with a symptom-limited Bruce treadmill exercise protocol or vasodilator protocol, as described previously $(10,11)$. For adenosine stress, adenosine was infused at $140 \mu \mathrm{g} / \mathrm{kg} / \mathrm{min}$ for $5 \mathrm{~min}$ and, when possible, low-level adjunctive treadmill exercise was performed during adenosine infusion (12). A 12-lead electrocardiogram was monitored continuously during stress testing. An ischemic stress electrocardiogram response of $\geq 1 \mathrm{~mm}$ horizontal or downsloping ST-segment depression or $\geq 1.5 \mathrm{~mm}$ upsloping was considered positive for ischemia.

Rest-Stress ${ }^{99 m}$ Tc-Sestamibi Protocol. For the rest-stress ${ }^{99 \mathrm{~m}} \mathrm{Tc}$-sestamibi protocol (5), a weight-adjusted dose of ${ }^{99 \mathrm{~m}} \mathrm{Tc}-$ sestamibi (296-333 MBq [8-9 mCi]) was injected at rest. A 4-min rest image was acquired with the patient upright, beginning at 30-60 min after injection, followed by a 4-min supine acquisition. Patients then underwent symptom-limited standard exercise treadmill testing or adenosine stress with injection of a weightadjusted dose of ${ }^{99 m} \mathrm{Tc}-$ sestamibi $(1,332-1,480 \mathrm{MBq}$ [36-40 $\mathrm{mCi}])$ at the time of peak stress. Beginning 15-30 min after stress injection, 2-min images were obtained with the patient first in the upright and then in the supine position (5).

Stress ${ }^{201}$ Tl-Rest ${ }^{99 m}$ Tc-Sestamibi Protocol. For the stress ${ }^{201} \mathrm{Tl}-$ rest ${ }^{99 \mathrm{~m}} \mathrm{Tc}-\mathrm{sestamibi}$ protocol $(8)$, patients were injected

TABLE 1

Characteristics of Patients with ICA

\begin{tabular}{|c|c|c|c|}
\hline Characteristic & Total $(n=56)$ & $\begin{array}{l}\text { Rest-stress }{ }^{99 \mathrm{~m} T c-\text { sestamibi }} \\
\qquad(n=39)\end{array}$ & $\begin{array}{c}\text { Stress }{ }^{201} \text { TI-rest }{ }^{99 m} \text { Tc-sestamibi } \\
\qquad(n=17)\end{array}$ \\
\hline Age $(y)$ & $64 \pm 13$ & $65 \pm 14$ & $62 \pm 12$ \\
\hline Men & $35(63)$ & $24(62)$ & $11(65)$ \\
\hline Body mass index $\left(\mathrm{kg} / \mathrm{m}^{2}\right)$ & $28 \pm 7$ & $29 \pm 7$ & $28 \pm 7$ \\
\hline Hypertension & $39(70)$ & $28(72)$ & $11(65)$ \\
\hline Diabetes & $14(25)$ & $10(26)$ & $4(24)$ \\
\hline Hypercholesterolemia & $29(52)$ & $20(51)$ & $9(53)$ \\
\hline Smoking & $6(11)$ & $3(8)$ & $3(18)$ \\
\hline Typical angina & $4(7)$ & $4(10)$ & $0(0)$ \\
\hline Atypical angina & $31(55)$ & $21(54)$ & $10(59)$ \\
\hline Shortness of breath & $5(9)$ & $2(5)$ & $3(18)$ \\
\hline Exercise stress & $25(45)$ & $17(44)$ & $8(47)$ \\
\hline Time between HS-SPECT and ICA (d) & $26 \pm 33$ & $23 \pm 34$ & $22 \pm 19$ \\
\hline
\end{tabular}

Data are mean $\pm \mathrm{SD}$ or $n$, with percentages in parentheses. 
TABLE 2

Characteristics of Patients with LLk of CAD

\begin{tabular}{|c|c|c|}
\hline Characteristic & Rest-stress ${ }^{99 m}$ Tc-sestamibi $(n=60)$ & Stress ${ }^{201}$ TI-rest ${ }^{99 m T c-s e s t a m i b i ~}(n=26)$ \\
\hline Age (y) & $56 \pm 13$ & $57 \pm 13$ \\
\hline Men & $30(50)$ & $11(42)$ \\
\hline Body mass index $\left(\mathrm{kg} / \mathrm{m}^{2}\right)$ & $28 \pm 7$ & $28 \pm 7$ \\
\hline Hypertension & $42(70)$ & $15(58)$ \\
\hline Diabetes & $11(18)$ & $6(23)$ \\
\hline Hypercholesterolemia & $29(48)$ & $14(54)$ \\
\hline Smoking & $2(3)$ & $2(8)$ \\
\hline Exercise stress & $31(52)$ & $15(58)$ \\
\hline
\end{tabular}

with ${ }^{201} \mathrm{Tl}$ at peak stress. The specific weight-based dose regimen used was to inject $74 \mathrm{MBq}(2 \mathrm{mCi})$ in patients $\leq 225 \mathrm{lbs}$ and 92.5 $\mathrm{MBq}(2.5 \mathrm{mCi})$ in patients $>225 \mathrm{lbs}$. Beginning approximately $10 \mathrm{~min}$ after exercise stress or $1 \mathrm{~min}$ after adenosine stress, a 6-min supine image was acquired, followed by a 6-min upright acquisition. Subsequently, without moving the patient from the imaging chair, ${ }^{99 \mathrm{~m}} \mathrm{Tc}$-sestamibi was injected $(296 \mathrm{MBq}$ [8 mCi] in patients $\leq 225 \mathrm{lbs}$ and $370 \mathrm{MBq}[10 \mathrm{mCi}]$ in patients $>225 \mathrm{lbs}$ ), and beginning 2 min after injection, a 4-min rest upright image was acquired, followed by a 4-min supine rest acquisition (8).

\section{HS-SPECT Scanner and Imaging Method}

The HS-SPECT system uses 9 collimated, pixilated CZT detector columns, mounted vertically in a $90^{\circ}$ geometry (2-4). Each of the columns consists of $1,024(16 \times 64) 5$-mm-thick CZT elements $(2.46 \times 2.46 \mathrm{~mm})$. Square-hole tungsten collimators are fitted to each of the detectors, with the size of the collimator holes matching the dimensions of the detector elements. Scintigraphic data are acquired by the detector columns rotating in synchrony, focusing on the region of interest (the heart), and are saved in list mode along with R-wave markers. Before imaging in both the upright and the supine positions, the detector was positioned parallel to the patient's chest, with care taken to align the heart in the center of the field of view. A 10-s prescan image was acquired at the beginning of each imaging session to identify the location of the heart within the chest and to set the angle limits of scanning (center of the region of interest) for each detector. Each image set was acquired with 120 projections per detector for the supine and upright positions. Transaxial images were generated using a proprietary Broadview reconstruction algorithm (Spectrum-Dynamics), based on the maximum-likelihood expectation maximization method (4). No attenuation or scatter correction was applied. Images were then reoriented into short-axis and vertical and horizontal long-axis slices using standard software (QPS; Cedars-Sinai Medical Center).

\section{Automated Quantification of SPECT Myocardial Perfusion Imaging (MPI)}

We have previously shown higher accuracy by combining supine and prone images than using supine images alone for the detection of CAD with conventional SPECT (7). Because prone imaging is not possible with HS-SPECT, we used both upright and supine imaging, with the supposition that soft-tissue attenuation or patient motion artifacts would potentially create attenuation artifacts in only 1 of the acquisitions or in separate regions of the myocardium and would hence allow us to distinguish artifacts from true perfusion defects. Upright and supine images were quantified separately using their respective upright and supine reference limits, and all MPS studies were quantitatively reanalyzed using automatic computer analysis (6). Automatically generated myocardial contours were evaluated by an experienced imaging cardiologist without knowledge of any clinical data, and when necessary, contours were adjusted to correspond to the myocardium (13\% of cases). The quantitative perfusion variable used was the TPD, which reflects a combination of both the defect severity and the extent of the defect in 1 parameter, as previously described (6). Stress perfusion defects on HS-SPECT were assessed by quantification of the TPD $(13,14)$.

Combined Upright-Supine Quantification. In addition to deriving separate TPD measures for upright TPD (U-TPD) and supine TPD (S-TPD), we applied a combined quantification approach to obtain upright-supine TPD (C-TPD) values. C-TPD is calculated by limiting the TPD computation from the supine polar map to pixels that have been quantified as abnormal on upright images (mean absolute deviation $>3.0$ ). The same threshold (mean absolute deviation $>3.0$ ) is used for supine images but only in locations determined to be abnormal on upright images; locations quantified as normal by upright imaging were not considered. Therefore, C-TPD represents the magnitude of perfusion defect seen on both supine and upright images in the same location and is analogous to the TPD derived from standard supine and prone images that we have previously described for conventional SPECT (7). The C-TPD parameter was expressed in the same units (percentage of the myocardium) as in the separate supine and upright TPD measurements. In determining the optimal cutoff for TPD, we considered the threshold for which $95 \%$ of the patients used to generate reference limits continued to remain in the normal perfusion category. The resultant thresholds of U-TPD $\geq 5 \%$, $\mathrm{S}$-TPD $\geq 5 \%$, and C-TPD $\geq 3 \%$ are in agreement with previously established thresholds for combined TPD quantification (7). For per-vessel analysis, we used the previously established threshold ( $\geq 2 \%$ for TPD measurements) $(6,15,16)$ for quantitative measurement in each coronary artery territory. Defects in the anterior and septal walls were allocated to the left anterior descending coronary artery; in the lateral wall to the left circumflex coronary artery; and in the inferior wall to the right coronary artery.

\section{ICA Analysis}

All invasive coronary angiograms were interpreted visually by experienced cardiologists who were unaware of MPS results. Patients did not have myocardial infarction or revascularization in 
the interval between MPS and ICA. A $\geq 70 \%$ luminal diameter stenosis was considered hemodynamically significant, except for the left main coronary artery, for which stenosis was defined as $\geq 50 \%$. Patients with left main CAD were considered to have stenosis in both the left anterior descending coronary and left circumflex coronary arteries.

\section{Statistical Analysis}

Statistical analyses were performed using software(Analyze-It, version 2.12; Analyze-It Software, Ltd.). All continuous variables were described as mean \pm SD. For all datasets, the Shapiro-Wilk test was used for normality. Datasets did not require any adjustment to convert them to a reference distribution. Unpaired $t$ tests were used to compare differences in unpaired continuous data for reference database variation, using $P<0.01$ as a cutoff. The receiver-operating-characteristic (ROC) curve analysis was performed to evaluate the ability of the quantification to predict $\geq 70 \%$ stenoses of coronary arteries. ROC curves were created using a step of $0.1 \%$ for the TPD values, and the differences between ROC curve areas (area \pm SE) were compared. Sensitivity and specificity between different types of acquisitions were compared using a paired McNemar test. In terms of the individual coronary territories, sensitivity and specificity were calculated at a cutoff point disease severity of $70 \%$ luminal diameter narrowing on ICA.

\section{RESULTS}

\section{Reference Limits}

The average count distributions derived from 30 male and 30 female patients with an LLk of CAD who underwent the stress $99 \mathrm{~m}$ Tc-sestamibi protocol are presented in Figure 1 , using a polar map display for men and women. Ranges of segmental average deviations in men were $5.1 \%-8.5 \%$ for upright, $5.3 \%-8.2 \%$ for supine, and $5.2 \%-8.4 \%$ for combined databases, and in women they were $5.9 \%-9.1 \%$ for upright, $5.8 \%-9.0 \%$ for supine, and $5.9 \%-8.8 \%$ for combined databases. Figure 2 shows the average count distri- butions derived from 15 men and 11 women with an LLk of CAD who underwent the stress ${ }^{201} \mathrm{Tl}$ protocol. Ranges of segmental average deviations in men were 6.0-8.6 for upright, $6.4 \%-8.3 \%$ for supine, and $6.6 \%-8.4 \%$ for combined databases, and in women they were $5.9 \%-9.4 \%$ for upright, $5.2 \%-10.0 \%$ for supine, and $5.7 \%-9.2 \%$ for combined databases. The normalized segmental myocardial uptake between upright and supine in any of the 17 segments for either men or women for either the ${ }^{201} \mathrm{Tl}$ or the 99m Tc-sestamibi protocol did not differ significantly. Because there were no significant differences between upright and supine limits, we have combined them and generated universal limits for the analysis of upright, supine, and combined data. These combined limits are shown in Figures 1 and 2 for rest-stress ${ }^{99 \mathrm{~m}}$ Tc-sestamibi and stress ${ }^{201} \mathrm{Tl}-$ rest ${ }^{99 \mathrm{~m}} \mathrm{Tc}$-sestamibi protocols. The combined upright-supine limits were subsequently used to identify perfusion defects (expressed as TPD) in the patients who underwent HS-SPECT and ICA.

\section{ICA Findings}

There were 34 patients with and 22 patients without obstructive coronary artery stenosis (Table 3). Regarding the distribution of significant stenoses, the numbers of left main, left anterior descending coronary artery, left circumflex coronary artery, and right coronary artery lesions were $3,28,15$, and 15 , respectively.

\section{TPD from Upright, Supine, and Combined Quantitative Analysis}

Of the 56 patients who underwent HS-SPECT and ICA, 40 patients had perfusion defects with U-TPD $\geq 5 \%$ on, 36 had defects with S-TPD $\geq 5 \%$, and 35 had defects with $\mathrm{C}$-TPD $\geq 3 \%$. Examples of combined upright-supine stress images of the rest-stress ${ }^{99 \mathrm{~m}} \mathrm{Tc}$-sestamibi protocol are shown in Figures $3 \mathrm{~A}$ and $3 \mathrm{~B}$. Figure $3 \mathrm{~A}$ shows a patient

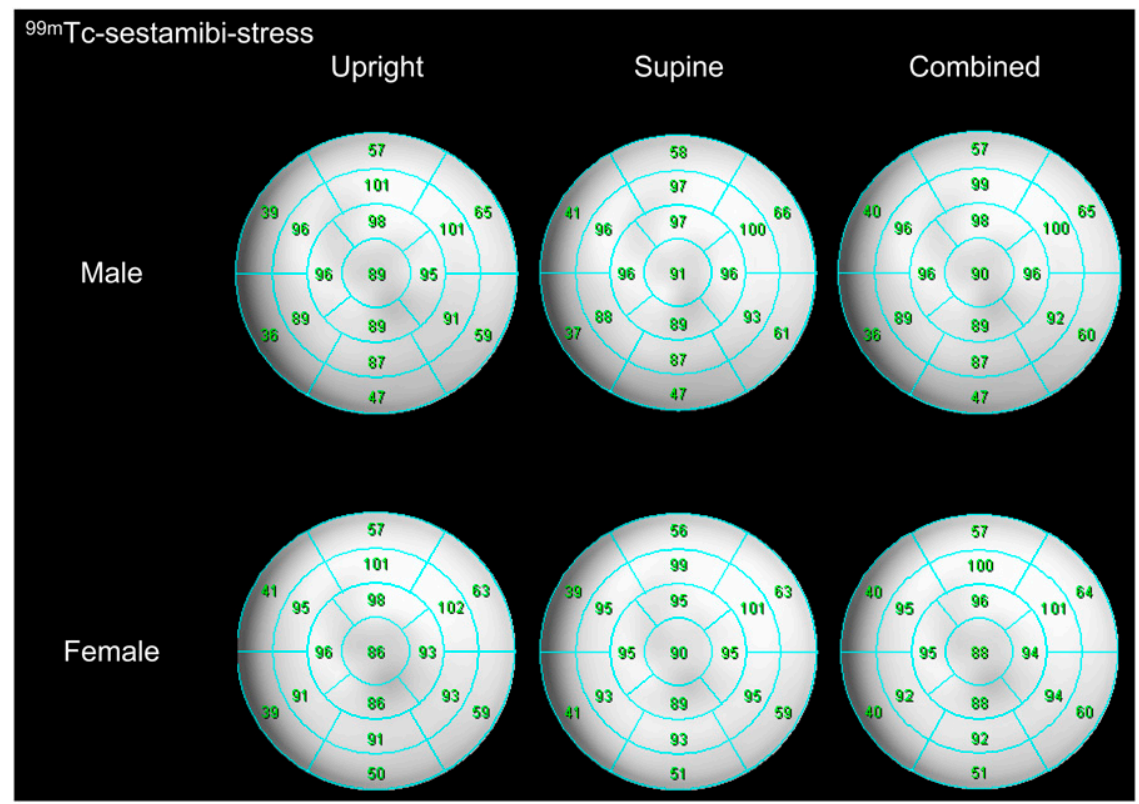

FIGURE 1. Polar maps for stress ${ }^{99 m T c-}$ sestamibi perfusion imaging of LLk men $(n=30)$ and women $(n=30)$ show segmental comparison of sex-dependent upright, supine, and combined mean reference perfusion distribution. Average myocardial uptake between supine and upright in any of 17 segments for either men or women did not differ significantly. 


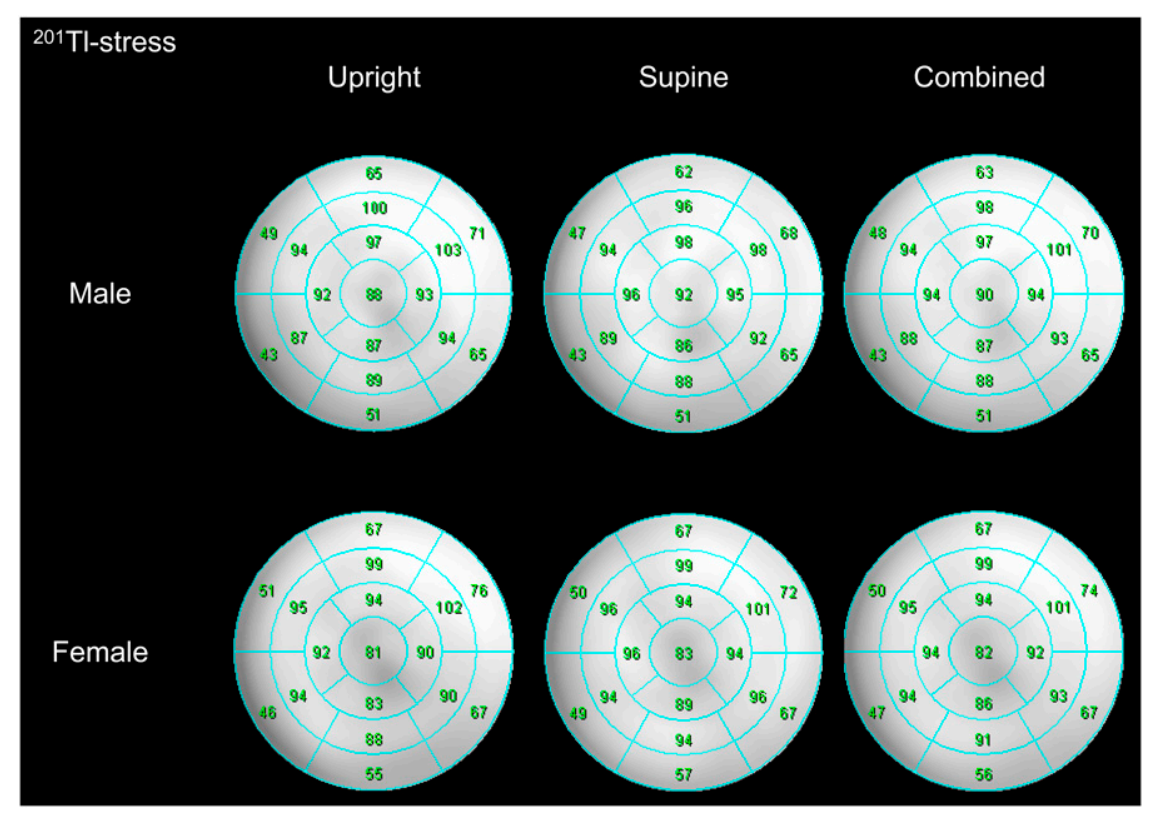

FIGURE 2. Polar maps for stress ${ }^{201} \mathrm{TI}$ perfusion imaging of LLk men $(n=15)$ and women $(n=11)$ show segmental comparison of sex-dependent upright, supine, and combined mean reference perfusion distribution. Average myocardial uptake between supine and upright in any of 17 segments for either men or women did not differ significantly.

with normal coronary angiography findings who demonstrated a basal-to-mid inferolateral wall defect on supine images and quantification and an inferolateral wall defect on prone images and quantification, with no significant defect detected by combined quantification. Figure 3B shows a patient with left anterior descending coronary artery disease who showed an anteroseptal wall defect on both supine and upright images that was detected by supine, upright, and combined quantification.

\section{ROC Curve Analysis}

ROC curves for the detection of CAD by TPD measurements derived from upright, supine, and combined uprightsupine datasets are shown in Figure 4. The areas under the ROC curves for U-TPD, S-TPD, and C-TPD were $0.88 \pm$ $0.05,0.89 \pm 0.04$, and $0.94 \pm 0.03$, respectively. The area under the ROC curve for C-TPD was significantly higher than that for U-TPD $(P<0.05)$. There was no significant difference in the area under the ROC curves between C-TPD and S-TPD or between U-TPD and S-TPD. As shown in Figure 5, the sensitivity was relatively unchanged among U-TPD, S-TPD, and C-TPD (not significant). On the other hand, specificity was significantly different among U-TPD,

TABLE 3

ICA Findings

\begin{tabular}{lc}
\hline \multicolumn{1}{c}{ Parameter } & No. of patients \\
\hline Maximum stenosis $<70 \%$ & 22 \\
Maximum stenosis $\geq 70 \%$ & 34 \\
Stenotic coronary arteries & \\
Left main & 3 \\
Left anterior descending & 28 \\
Left circumflex & 15 \\
Right coronary & 15 \\
\hline
\end{tabular}

S-TPD, and C-TPD ( $P=0.02$ for C-TPD vs. S-TPD, and $P<0.0001$ for C-TPD vs. U-TPD). When the analysis was performed separately for 2 different MPS protocols, the sensitivity was $92 \%, 92 \%$, and $92 \%$ for U-TPD, S-TPD, and C-TPD, and specificity was $73 \%, 73 \%$, and $87 \%$ for U-TPD, S-TPD, and C-TPD, respectively, with the rest-stress ${ }^{99 \mathrm{~m}} \mathrm{Tc}$-sestamibi protocol. The sensitivity was $100 \%, 80 \%$, and $100 \%$ for U-TPD, S-TPD, and C-TPD, and specificity was $57 \%, 71 \%$, and $86 \%$ for U-TPD, S-TPD, and C-TPD with the stress ${ }^{201}$ Tl-rest 99m Tc-sestamibi protocol.

\section{Per-Vessel Analysis}

On a per-vessel basis, using a TPD of $\geq 2 \%$ for the detection of significant stenosis, the sensitivity and specificity were $67 \%$ and $91 \%$ with upright images, $66 \%$ and $94 \%$ with supine images, and $69 \%$ and $97 \%$ with combined upright-supine images. The sensitivity was relatively unchanged among U-TPD, S-TPD, and C-TPD (not significant), although specificity was significantly different between U-TPD and C-TPD $(P=0.02)$.

\section{DISCUSSION}

This is the first report, to our knowledge, evaluating the diagnostic accuracy of HS-SPECT MPI for the detection of hemodynamically significant coronary artery stenosis, compared with ICA. In addition, it is the first report of the use of upright-supine imaging for these types of new HS-SPECT devices and the first report of combined automated quantification of such data as a method for eliminating attenuation artifacts related to patient positioning. We observed that with the combined upright-supine approach, the sensitivity for detection of CAD was $94 \%$ and that specificity for the absence of CAD was $86 \%$. For individual 


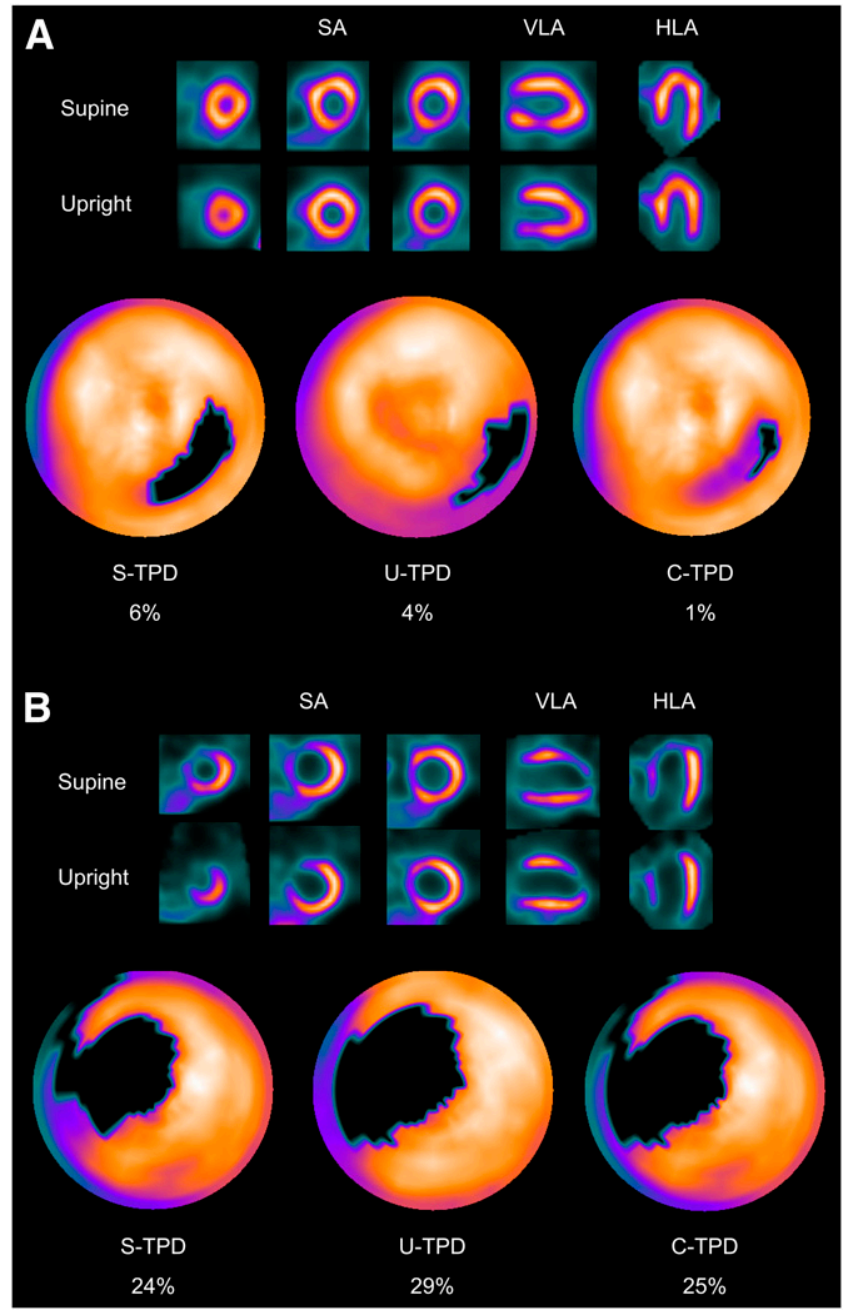

FIGURE 3. (A) Example of inferolateral wall supine and upright MPS artifact images from 49-y-old woman with typical chest pain and known hypertension, diabetes, hypercholesterolemia, and family history of premature CAD. Images are displayed in 3 short-axis views and in vertical and horizontal long-axis views. Electrocardiogram response to exercise was nonischemic for ST-segment depression. Poststress supine and upright images show reduced uptake of radiotracer in inferolateral wall (top and middle rows). Blackout maps (bottom row) give quantification results of $4 \%$ for U-TPD and 6\% for S-TPD. When supine and upright images are combined, only a small defect is visualized on black-out map, with C-TPD of $1 \%$. Subsequent coronary angiogram showed no significant stenosis. (B) Example of true anteroseptal wall defect on supine and upright MPS images from 43-y-old man with typical chest pain and known hypercholesterolemia. Electrocardiogram response to exercise was ischemic for ST-segment depression, and subsequent coronary angiogram revealed severe stenosis of proximal left anterior descending coronary artery. Both supine and upright images show apparent anteroseptal wall perfusion abnormality, and U-TPD, S-TPD, and C-TPD are 29\%, 24\%, and 25\%, respectively. Defects are visualized on black-out maps. HLA = horizontal long axis; $\mathrm{SA}=$ short axis; VLA $=$ vertical long axis.

coronary territories, we found a sensitivity and specificity of $70 \%$ and $97 \%$, respectively. The results were comparable to those found using the rest-stress ${ }^{99 \mathrm{~m}} \mathrm{Tc}$-sestamibi or stress ${ }^{201} \mathrm{Tl}-$ rest ${ }^{99 \mathrm{~m}} \mathrm{Tc}$-sestamibi protocols. These findings

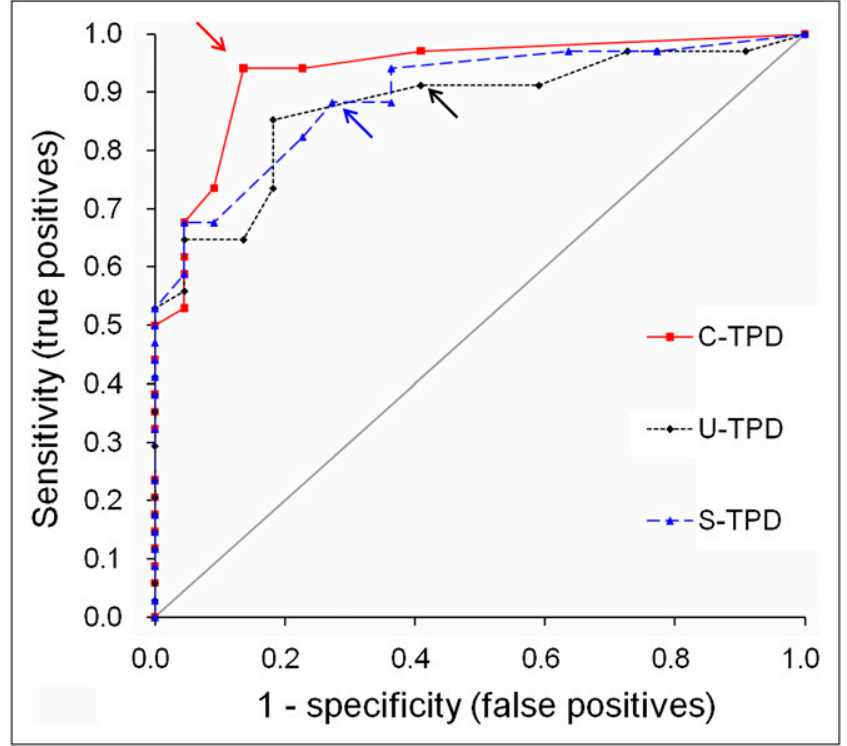

FIGURE 4. ROC curves for detection of CAD by measurements of U-TPD, S-TPD, and C-TPD in angiographic population $(n=56)$. Percentage TPD was compared with presence or absence of hemodynamically significant CAD as observed by ICA. U-TPD $\geq 5 \%$, S-TPD $\geq 5 \%$, and C-TPD $\geq 3 \%$ were used as cutoff points.

compare favorably with the results of MPS using the conventional Anger scintillation camera.

\section{Comparison with Prior Studies}

There is extensive literature on the diagnostic accuracy of conventional stress MPS in comparison with ICA. The overall sensitivity of exercise and vasodilator stress perfusion SPECT for the detection of angiographically significant ( $\geq 50 \%$ stenosis) CAD was $87 \%$ and $89 \%$, respectively, and specificity was $73 \%$ and $75 \%$, respectively (17). Prior studies that applied the automated TPD quantification of our study to Anger-camera MPS found an $85 \%$ sensitivity and $65 \%$ specificity for supine imaging (area under the ROC curve, $0.86 \pm 0.02$ ) and an $85 \%$ sensitivity and $86 \%$ specificity for combined supine-prone imaging (area under the ROC curve, $0.90 \pm 0.02$ ). Our results with HS-SPECT are

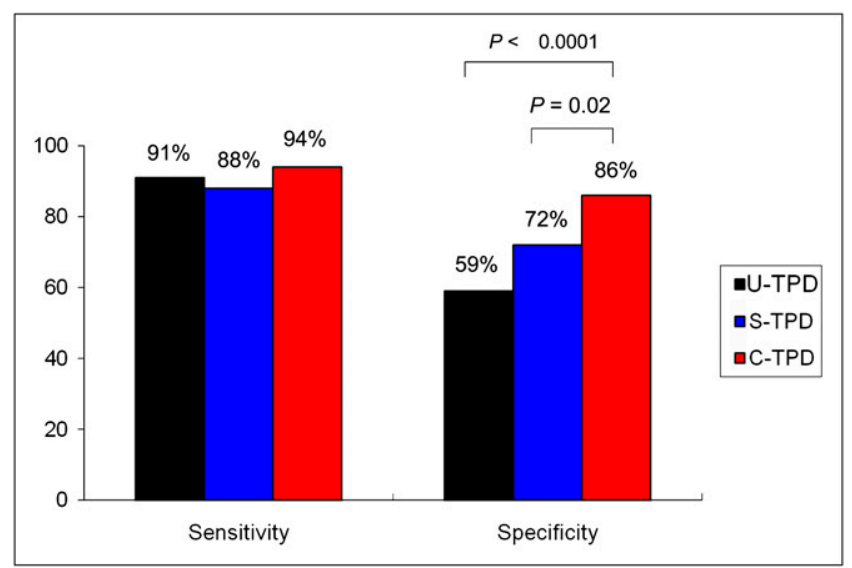

FIGURE 5. Sensitivities and specificities for detection of CAD by U-TPD, S-TPD, and C-TPD in angiographic population $(n=56)$. 
similar to the results of these prior studies (7). On a pervessel basis, prior studies have reported that the sensitivity and specificity were $79 \%$ and $76 \%$, respectively, with supine images (6). Our results are comparable to these findings.

\section{Quantitative Analysis}

We used quantitative analysis for the interpretation of the MPS studies. Quantitative analysis eliminates observer variability and bias and has been shown to be useful in comparing sequential MPI studies $(13,18)$ and assessing the effectiveness of invasive and medical treatments in patients with CAD (14). Quantitative analysis also provides an approach that might be more generalizable to other centers not dependent on the expertise of the interpreter for analysis with the new $\gamma$-camera.

\section{Upright and Supine Imaging}

We have previously shown that detection of CAD is more accurate with combined supine and prone MPS than with supine imaging alone. With the HS-SPECT system, prone imaging is not possible. We initially introduced the combined upright and supine imaging protocol when comparing the 2 stress ${ }^{201}$ Tl-rest ${ }^{99 m}$ Tc-sestamibi and rest-stress ${ }^{99 \mathrm{~m}} \mathrm{Tc}$-sestamibi protocols for the new fast cameras; however, these image pairs were analyzed only visually in that study (8). In comparison to prone-supine imaging using conventional cameras, the combined upright-supine imaging may be more practical using the new HS-SPECT devices, because the imaging times are much shorter. In addition, we have found that all patients tolerated the upright-supine, whereas some patients cannot tolerate prone imaging.

We have previously developed automated combined supine-prone analysis (7) and showed increased specificity for the diagnosis of CAD by that method. Here, the same concept of requiring the pixel location at both locations to be abnormal has been applied to the analysis of uprightsupine data. However, in contrast to the difference in reference limits for prone and supine data, we have shown that the reference limits are similar for upright and supine data (7), likely because of the lower variability between upright and supine positions. Therefore, we were able to use the same (combined) reference limits for both upright and supine but still required the abnormality to be present on both scans in the same location. This technique allows avoidance of artifacts, which can be present on one or the other scan and result in false-positive quantification. Naturally, reference thresholds obtained by such analysis are lower than those obtained in separate analysis because defect collocation is not perfect. With the lower abnormality threshold, the sensitivity of the test is preserved while the specificity is significantly improved.

\section{Study Limitations}

The study was performed at a single center, and whether our findings may be applicable to other centers will require further investigation; however, the use of automated quantitative analysis increases the likelihood that the results of our study could be replicated elsewhere. Data from 2 different protocols, rest-stress ${ }^{99 \mathrm{~m}} \mathrm{Tc}$-sestamibi and stress ${ }^{201} \mathrm{Tl}$-rest ${ }^{99 \mathrm{~m}} \mathrm{Tc}$-sestamibi MPS, were combined; however, there was no significant difference in the sensitivity and specificity of HS-SPECT when these datasets were analyzed individually or when they were combined. Our study was a retrospective analysis of patients who had undergone HS-SPECT and ICA within 6 mo. Although this duration between studies was fairly long, the mean duration between studies was $23 \mathrm{~d}$ and $73 \%$ of patients underwent both studies within 4 wk. Moreover, patients considered for analysis did not have a change in clinical status between the studies. The ICA was performed for clinical purposes. This practice results in a selection bias, because patients with abnormal SPECT findings are more likely to be referred for ICA. The number of studies was limited because of the novelty of the imaging protocols; however, significant differences were demonstrated in the specificity and increased area of the ROC curve for the combined (upright and supine) analysis. The exact cutoff point for optimal specificity is difficult to establish because of the few angiographically normal cases $(n=22)$, and we chose to use an LLk population to establish the reference thresholds. However, in this study we showed that the performance of combined imaging is improving the overall diagnostic performance as measured by the area under the ROC curve. Specific cutoff thresholds for a given protocol may need to be confirmed in larger studies. The exact mechanism by which test performance is improved through combining supine with upright information is not currently known and warrants further investigation.

\section{CONCLUSION}

In this first report, to our knowledge, comparing HSSPECT with ICA, automatic quantitative HS-SPECT shows high diagnostic accuracy for detecting clinically significant CAD, with findings comparable to those reported using conventional SPECT. Similar to findings with supine and prone imaging on conventional SPECT cameras, combined upright and supine acquisitions with HS-SPECT eliminates imaging artifacts present on 1 type of scan and leads to increased specificity of HS-SPECT in detecting CAD.

\section{ACKNOWLEDGMENTS}

This research was supported in part by grant R0HL089765-01 from the National Heart, Lung, and Blood Institute/National Institutes of Health (NHLBI/NIH). The contents of this report are solely the responsibility of the authors and do not necessarily represent the official views of the NHLBI. Drs. Daniel Berman and Piotr Slomka participate in software royalties at Cedars-Sinai Medical Center. Dr. Daniel Berman is a shareholder in SpectrumDynamics. 


\section{REFERENCES}

1. Slomka PJ, Patton JA, Berman DS, Germano G. Advances in technical aspects of myocardial perfusion SPECT imaging. J Nucl Cardiol. 2009;16:255-276.

2. Patton J, Slomka P, Germano G, Berman DS. Recent technological advances in nuclear cardiology. J Nucl Cardiol. 2007;14:501-513.

3. Sharir T, Ben-Haim S, Merzon K, et al. High-speed myocardial perfusion imaging: initial clinical comparison with conventional dual detector Anger camera imaging. JACC Cardiovasc Imaging. 2008;1:156-163.

4. Gambhir SS, Berman DS, Ziffer J, et al. A novel high-sensitivity rapid-acquisition single-photon cardiac imaging camera. J Nucl Med. 2009;50:635-643.

5. Sharir T, Slomka PJ, Hayes SW, et al. Multicenter trial of high-speed versus conventional SPECT imaging: quantitative results of myocardial perfusion and left ventricular function. J Am Coll Cardiol. 2010;55:1965-1974.

6. Slomka PJ, Nishina H, Berman DS, et al. Automated quantification of myocardial perfusion SPECT using simplified normal limits. J Nucl Cardiol. 2005;12:66-77.

7. Nishina H, Slomka PJ, Abidov A, et al. Combined supine and prone quantitative myocardial perfusion SPECT: method development and clinical validation in patients with no known coronary artery disease. J Nucl Med. 2006;47:51-58.

8. Berman DS, Kang X, Tamarappoo B, et al. Stress thallium-201/rest technetium$99 \mathrm{~m}$ sequential dual isotope high-speed myocardial perfusion imaging. JACC Cardiovasc Imaging. 2009;2:273-282.

9. Diamond GA, Forrester JS. Analysis of probability as an aid in the clinical diagnosis of coronary artery disease. N Engl J Med. 1979;300:1350-1358.

10. Berman DS, Germano G. Interpretation and reporting of gated myocardial perfusion SPECT. In: Germano G, Berman DS, eds. Clinical Gated Cardiac SPECT. 2nd ed. Oxford, U.K.: Blackwell Publishing; 2006:139-171.
11. Berman DS, Abidov A, Kang X, et al. Prognostic validation of a 17-segment score derived from a 20-segment score for myocardial perfusion SPECT interpretation. J Nucl Cardiol. 2004;11:414-423.

12. Berman DS, Kang X, Hayes SW, et al. Adenosine myocardial perfusion singlephoton emission computed tomography in women compared with men: impact of diabetes mellitus on incremental prognostic value and effect on patient management. J Am Coll Cardiol. 2003;41:1125-1133.

13. Berman DS, Kang X, Gransar H, et al. Quantitative assessment of myocardial perfusion abnormality on SPECT myocardial perfusion imaging is more reproducible than expert visual analysis. $J$ Nucl Cardiol. 2009;16:45-53.

14. Shaw LJ, Berman DS, Maron DJ, et al. Optimal medical therapy with or without percutaneous coronary intervention to reduce ischemic burden: results from the Clinical Outcomes Utilizing Revascularization and Aggressive Drug Evaluation (COURAGE) trial nuclear substudy. Circulation. 2008;117:1283-1291.

15. Berman DS, Kang X, Slomka PJ, et al. Underestimation of extent of ischemia by gated SPECT myocardial perfusion imaging in patients with left main coronary artery disease. J Nucl Cardiol. 2007;14:521-528.

16. Xu Y, Fish M, Gerlach J, et al. Combined quantitative analysis of attenuation corrected and non-corrected myocardial perfusion SPECT: method development and clinical validation. $\mathrm{J} \mathrm{Nucl} \mathrm{Cardiol}$. In press.

17. Klocke FJ, Baird MG, Lorell BH, et al. ACC/AHA/ASNC Guidelines for the clinical use of cardiac radionuclide imaging: executive summary-a report of the American College of Cardiology/American Heart Association Task Force on Practice Guidelines (ACC/AHA/ASNC Committee to Revise the 1995 Guidelines for the Clinical Use of Cardiac Radionuclide Imaging. Circulation. 2003;108:1404-1418.

18. Slomka PJ, Berman DS, Germano G. Quantification of serial changes in myocardial perfusion. J Nucl Med. 2004;45:1978-1980. 